\title{
The surface tension and interfacial composition of water/ethanol mixture
}

\author{
Chi M. Phan* \\ Discipline of Chemical Engineering, Curtin University, Perth, WA 6845, Australia \\ *Corresponding email: c.phan@ curtin.edu.au
}

\begin{abstract}
This study develops a model to fit the surface tension of the water/ethanol mixture for the complete composition range from 0 to 1 . The theoretical framework complements Van Der Waals's theory on the density gradient. The modelling results overcome the limitation of Gibbs adsorption isotherm, which completely ignores the composition of the interfacial layer. Furthermore, the model presents a new method to correctly calculate the composition of the interfacial layer for binary liquid mixture and surfactant solutions.
\end{abstract}

Keywords: surface tension, ethanol, density gradient theory

\section{Preamble}

The nature of the surface tension has fascinated many generations of scientists. In 1830, Gauss summarised the earlier works of Young and Laplace to describe the surface tension via the difference in density. The Young-Laplace-Gauss equation treats the interface between two fluids as a static surface with a zero thickness, also known as a capillary surface [1]. However, the static theory cannot describe the interfacial layer, which is central to surface science. By the $19^{\text {th }}$ century, it was well accepted that liquid molecules are in a rapid and steady state of motion. As a result, the thermodynamic equilibrium is developed to relate the surface energy, or the surface tension, to liquid composition. One of the critical advances of the thermodynamic model is the Gibbs adsorption isotherm [2], which predicts the change of surface tension from the bulk concentration. Gibbs's equation reduces the continuous transition between liquid and vapour phases to a discontinuity, defined as a dividing plane. Thus, the equation becomes an irreplaceable basis of surfactant studies [2]. However, the equation cannot fit the surface tension of water/alcohol mixtures, which are miscible for the entire composition range $[3,4]$. The shortcoming of Gibbs adsorption was evidenced by non-aqueous binary mixtures [5]. The equation also leads to controversies around the saturated adsorption of surfactants [6]. For the 
water/ethanol mixture, the usage of the equation led to a conflicting interpretation of the interfacial thickness [7-9].

Shortly after Gibbs's proposal, Van der Waals developed an alternative treatment of the thermodynamic interface with a finite thickness and continuously variable density [10]. The theory, commonly known as "density gradient theory", is one of the few available tools to quantify the properties of liquid surface [11]. On the other hand, the density-based model is too complicated to describe the surface tension effectively [12]. Recently, we have experimentally investigated the composition of the surface layer for water/deuterated ethanol mixtures. Although the results were only obtainable for low concentrations (due to the limitation of the neutron reflectometry [7]), it was found that the surface tension follows the linear interpolation of the interfacial composition [13,14]. This short communication presents a model to describe the water/ethanol surface tension for the complete composition range. The successful model can effectively calculate the surface energy via interfacial composition without using complicated density gradients.

\section{Theoretical model and results}

The surface tension of the liquid mixture is given by linear interpolation of the molar composition of the interfacial layer, as proposed by Eberhart [13]. The rule has been validated for water/ethanol by neutron reflectometry [7] and simulations [14]. The surface tension of water/ethanol for a given bulk molar fraction, $x$, is given by:

$$
\gamma(x)=\gamma_{\mathrm{a}} S_{\mathrm{a}}+\gamma_{\mathrm{w}}\left[1-S_{a}\right]
$$

Where $S_{\mathrm{a}}$ is the ethanol molar composition of the interfacial layer, $\gamma(x), \gamma_{\mathrm{a}}$ and $\gamma_{\mathrm{w}}$ are the surface tension of mixture, pure ethanol and pure water, respectively.

This model aims to relate $S_{a}$ to the bulk molar fraction, $x$. Since the adsorption is required for the entire composition, from 0 to 1 , a sigmoidal isotherm is used [15]:

$$
\begin{aligned}
& S_{S}=\frac{1}{D}\left\{\arctan \left(\frac{x-A}{B}\right)-\arctan \left(\frac{-A}{B}\right)\right\} \\
& D=\arctan \left(\frac{1-A}{B}\right)-\arctan \left(\frac{-A}{B}\right)
\end{aligned}
$$

Where $A$ and $B$ are the dimensionless adsorption parameters. 
Equation (2) was derived from a distribution function of adsorbed molecules, in which the adsorbate-adsorbate interaction dominates the adsorbent-adsorbate interaction. The value of $A$ represents the locus of the distribution, and $B$ represents the width of the distribution [15].

In contrast to the conventional analysis [16], the model considers the interfacial layer as a separate region with a non-zero thickness and distinguishing composition. As the interfacial region has a uniform composition, the surface coverage, $S_{s}$, can be assumed as the volume fraction of ethanol in the interfacial layer. The value of $S_{a}$ in Eq.(1) can be directly related to $S_{s}$ via the molecular volume of water and ethanol:

$$
S_{a}=\frac{S_{s}}{V_{a}} /\left(\frac{S_{s}}{V_{a}}+\frac{1-S_{S}}{V_{w}}\right)
$$

Where $V_{\mathrm{a}}$ and $V_{\mathrm{w}}$ are the molar volume of ethanol and water, respectively.

At $25^{\circ} \mathrm{C}$, the values of $V_{\mathrm{a}}$ and $V_{\mathrm{w}}$ can be calculated from the densities [17] as 58.67 and 18.07 $\mathrm{cm}^{3} / \mathrm{mol}$. Similarly, $\gamma_{\mathrm{a}}(22.02 \mathrm{mN} / \mathrm{m})$ and $\gamma_{\mathrm{w}}(71.89 \mathrm{mN} / \mathrm{m})$ are readily obtained from the experimental tension $[3,18]$. The above equations can be fitted to experimental data of $\gamma$ versus $x$ (Figure 1).

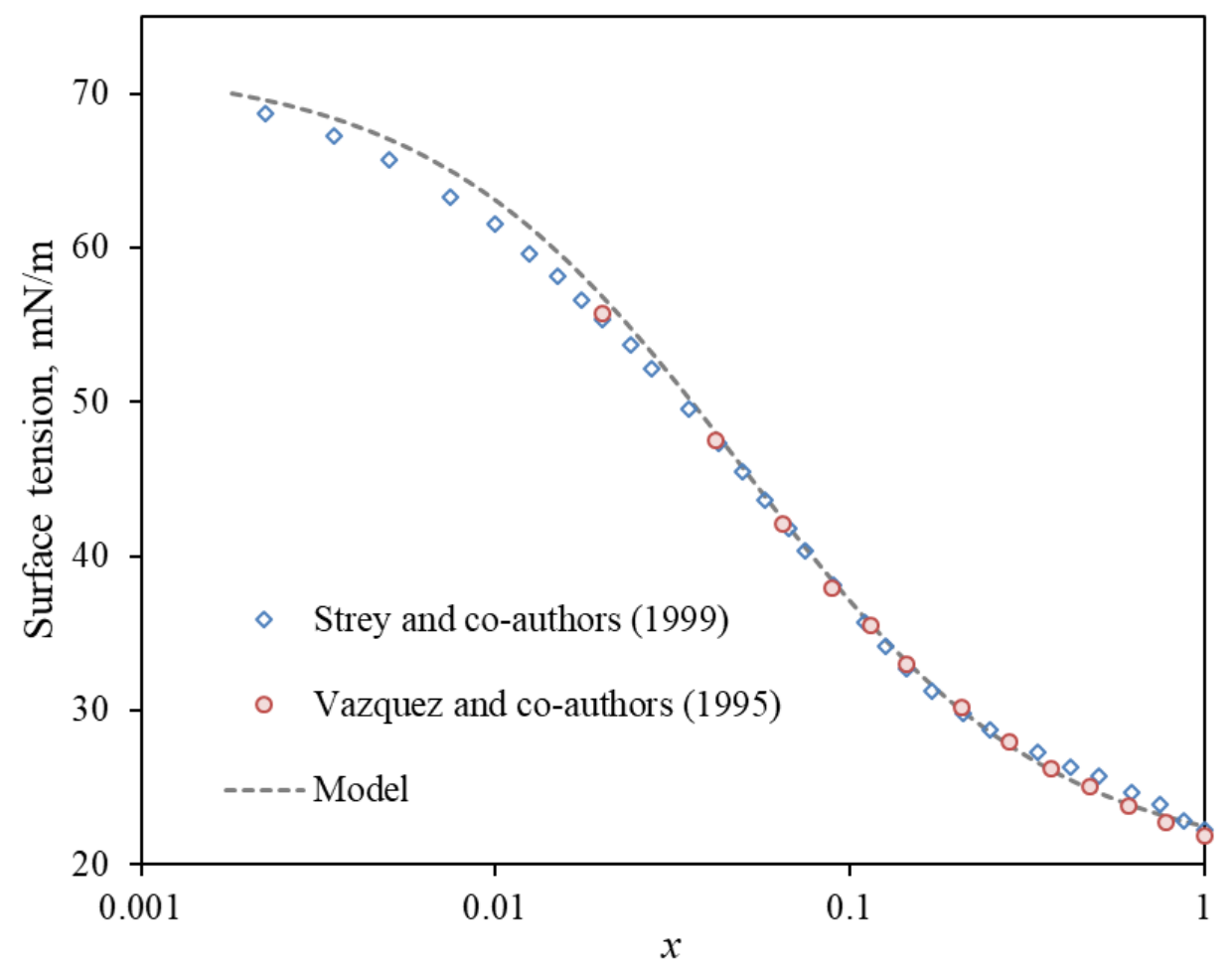

Figure 1. Modelling surface tension of water/ethanol $\left(A=-1.46 \times 10^{-2}, B=5.00 \times 10^{-7}\right)$. Experimental data were collected from the literature values $[3,18]$. 
It can be seen that the new model can fit the surface tension for the whole composition range from 0 to 1 . The simplicity of the model overcomes the complexity and limitation of the Gibbs adsorption isotherm. As discussed by many researchers, the Gibbs adsorption is based on the activity $a_{s}$, not a molar fraction. The issue with the conventional method is that the activity coefficient (that is, the ratio between activity and molar fraction) is not constant. Consequently, a variable activity coefficient is required. Furthermore, the application of the Gibbs isotherm indicated a failure above the apparent "critical micelle concentration", at $x \sim 0.02$ (or $a_{s} \sim 0.1$ ) in Figure 2 [3]. Similarly, Yano applied the modified partial pressure of alcohol and found a critical shift of activity at $x \sim 0.06$ [4]. In most cases, the conventional methods require at least three parameters. In contrast, this work avoids the obstacle around the activity coefficient by using the distribution adsorption equation (Eq.(2)). The model in Figure 1 provides a smooth transition, with two adjustable parameters, over the entire composition range.

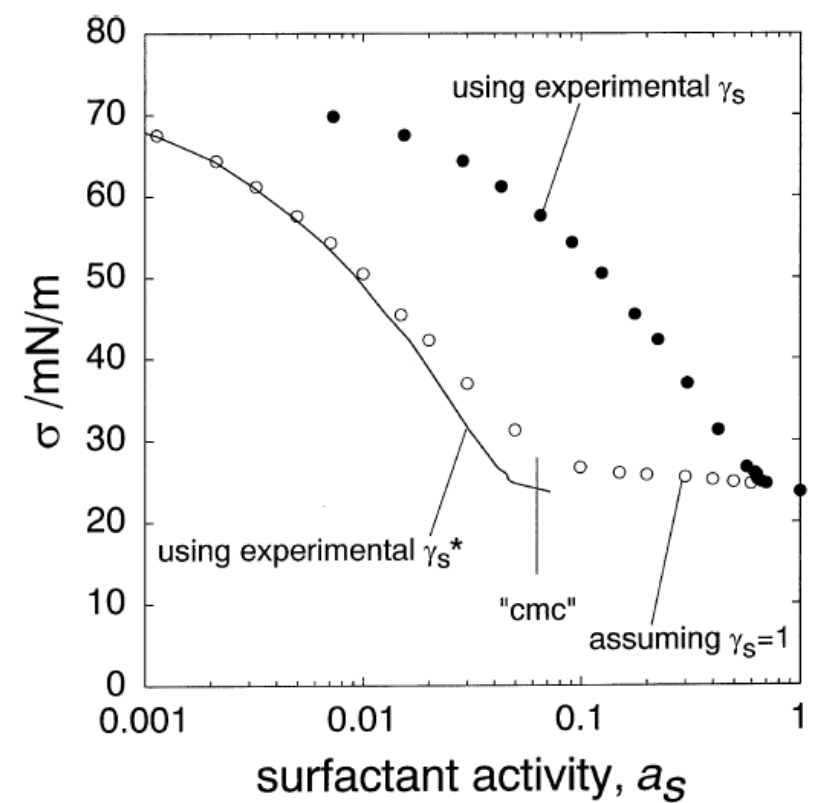

Figure 2. Surface tension of water/ethanol mixtures using the Gibbs adsorption isotherm [3]. In this figure: $\gamma_{s}$ is the activity coefficient, and $a_{S}$ is the activity. Reprinted with permission from reference 3. Copyright 1999 American Chemical Society.

One of the potential expansions of the equation is to redefine $A$ and $B$ to include the temperature [15]. Furthermore, the new model can be extended to the surface tension of other binary mixtures. For example, the modelling of water/propanol is showed in Figure 3. Similarly, the solution of non-ionic surfactants can be considered as a binary mixture of the solvated surfactant and free water. The molecular volume, $V_{\mathrm{a}}$, of solvated surfactants should be calculated from the solubility [19]. The value of $\gamma_{\mathrm{a}}$ can be estimated from the saturated surface tension or the hypothetical tension of a pure solute, as proposed by Wexley and Dutcher [20]. 
For ionic surfactants, the model needs to consider the surfactant and counter-ion separately [21]. The comprehensive extension is under development.

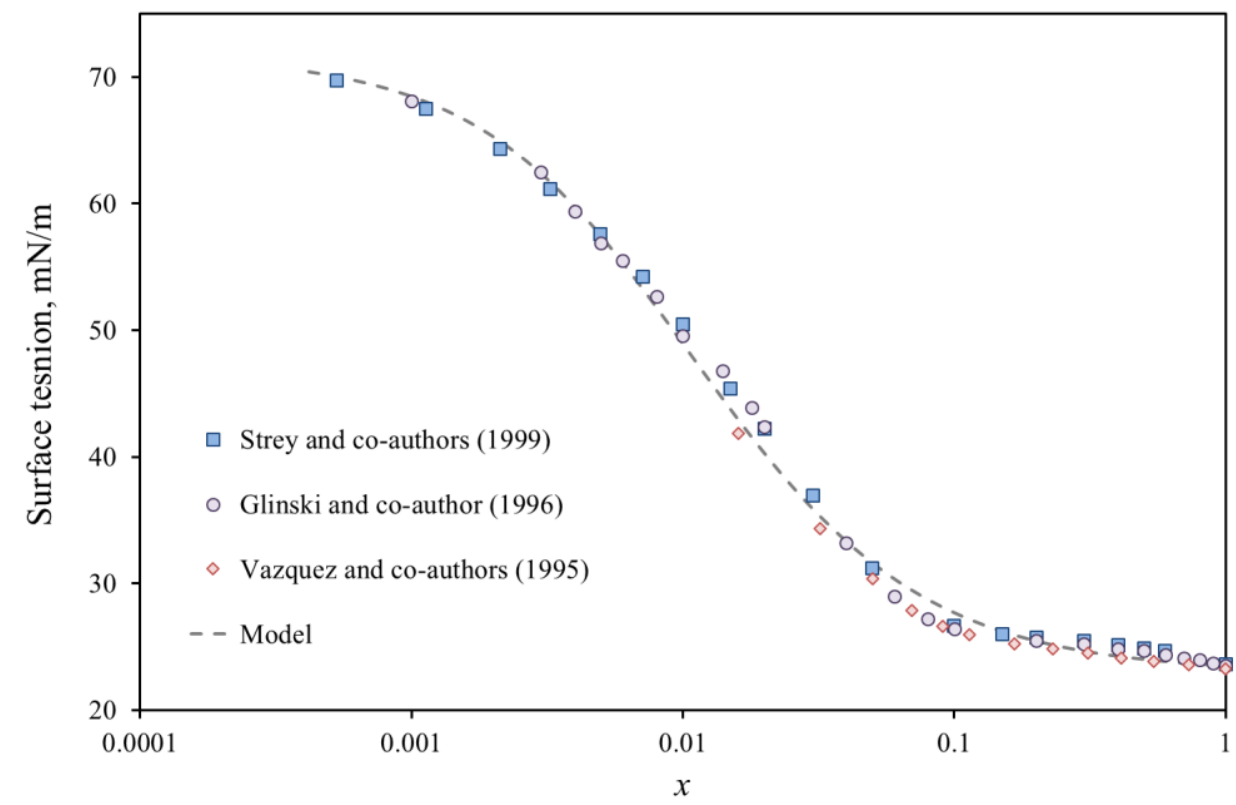

Figure 3. Modelling surface tension of water/n-propanol at $25^{\circ} \mathrm{C}\left(A=-1.41 \times 10^{-3}, B=2.75 \times 10^{-}\right.$ $\left.{ }^{3}\right)$. Experimental data were collected from the literature values $[3,18,22]$.

After more than a century since the Van der Waals proposal for the density composition of a finite transition layer, it is demonstrated the composition of such layer can be used to quantify the surface energy correctly. The proposed model fits surface energy to the surface composition without a complicated computational scheme [12]. The model provides an important foundation to treat the surface of surfactant solutions as a real fluid interface instead of a capillary surface $[1]$.

\section{References}

[1] R. Finn, Capillary surface interactions, Not. AMS. 46 (1999) 770-781.

[2] M.J. Rosen, J.T. Kunjappu, Surfactants and interfacial phenomena, Wiley, 2012.

[3] R. Strey, Y. Viisanen, M. Aratono, J.P. Kratohvil, Q. Yin, S.E. Friberg, On the necessity of using activities in the Gibbs equation, J. Phys. Chem. B. 103 (1999) 91129116. doi:10.1021/jp990306w.

[4] Y.F. Yano, Correlation between surface and bulk structures of alcohol-water mixtures, J. Colloid Interface Sci. 284 (2005) 255-259. doi:10.1016/j.jcis.2004.09.059.

[5] S. Stephan, H. Hasse, Enrichment at vapour-liquid interfaces of mixtures: establishing a link between nanoscopic and macroscopic properties, Int. Rev. Phys. Chem. 39 (2020) 319-349. doi:10.1080/0144235X.2020.1777705.

[6] F.M. Menger, L. Shi, S.A.A. Rizvi, Re-evaluating the Gibbs analysis of surface tension at the air/water interface, J. Am. Chem. Soc. 131 (2009) 10380-10381. doi:10.1021/ja9044289. 
[7] A.E. Hyde, M. Ohshio, C. V. Nguyen, S. Yusa, N.L. Yamada, C.M. Phan, Surface properties of the ethanol/water mixture: Thickness and composition, J. Mol. Liq. 290 (2019) 111005. doi:10.1016/j.molliq.2019.111005.

[8] A. Bagheri, M. Fazli, M. Bakhshaei, Surface properties and surface thickness of aqueous solutions of alcohols, J. Mol. Liq. 224 (2016) 442-451. doi:10.1016/j.molliq.2016.09.113.

[9] M.S.C.S. Santos, J.C.R. Reis, Thermodynamic evaluation of molar surface area and thickness of water + ethanol mixtures, J. Mol. Liq. 255 (2018) 419-428. doi:10.1016/j.molliq.2018.01.136.

[10] J.S. Rowlinson, Translation of J. D. van der Waals" "The thermodynamik theory of capillarity under the hypothesis of a continuous variation of density," J. Stat. Phys. 20 (1979) 197-200. doi:10.1007/BF01011513.

[11] S. Stephan, K. Langenbach, H. Hasse, Enrichment of components at vapour - Liquid interfaces: A study by molecular simulation and density gradient theory, Chem. Eng. Trans. 69 (2018) 295-300. doi:10.3303/CET1869050.

[12] X. Liang, M.L. Michelsen, G.M. Kontogeorgis, Pitfalls of using the geometric-mean combining rule in the density gradient theory, Fluid Phase Equilib. 415 (2016) 75-83. doi:10.1016/j.fluid.2016.01.047.

[13] J.G. Eberhart, The surface tension of binary liquid mixtures, J. Phys. Chem. 70 (1966) 1183-1186. doi:10.1021/j100876a035.

[14] C.M. Phan, C.V. Nguyen, T.T.T. Pham, Molecular Arrangement and Surface Tension of Alcohol Solutions, J. Phys. Chem. B. 120 (2016). doi:10.1021/acs.jpcb.6b01209.

[15] J.J. Mahle, An adsorption equilibrium model for Type 5 isotherms, Carbon. 40 (2002) 2753-2759. doi:10.1016/S0008-6223(02)00201-4.

[16] C.A. Eckert, J.M. Prausnitz, Statistical surface thermodynamics of simple liquid mixtures, AIChE J. 10 (1964) 677-683. doi:10.1002/aic.690100520.

[17] J. Ortega, Densities and Refractive Indices of Pure Alcohols as a Function of Temperature, J. Chem. Eng. Data. 27 (1982) 312-317. doi:10.1021/je00029a024.

[18] G. Vazquez, E. Alvarez, J.M. Navaza, Surface Tension of Alcohol + Water from 20 to $50{ }^{\circ} \mathrm{C}$, J. Chem. Eng. Data. 40 (1995) 611-614. doi:10.1021/je00019a016.

[19] W.N. Maclay, Factors affecting the solubility of nonionic emulsifiers, J. Colloid Sci. 11 (1956) 272-285. doi:10.1016/0095-8522(56)90052-6.

[20] A.S. Wexler, C.S. Dutcher, Statistical mechanics of multilayer sorption: Surface tension, J. Phys. Chem. Lett. 4 (2013) 1723-1726. doi:10.1021/jz400725p.

[21] C.M. Phan, Dissociation of Ionic Surfactants at the Air/Water Interface: Complete or Partial?, J. Phys. Chem. B. 120 (2016) 7681-7686. doi:10.1021/acs.jpcb.6b06074.

[22] J. Glinski, G. Chavepeyer, J.K. Platten, Surface properties of diluted aqueous solutions of normal propyl alcohol, J. Chem. Phys. 104 (1996) 8816-8820. doi:10.1063/1.471571. 